\title{
An in vitro model of hepatic steatosis using lipid loaded induced pluripotent stem cell derived hepatocyte like cells
}

Hiraganahalli Bhaskar Deepak, Nellikalaya Shreekrishna, Zaheerbasha Sameermahmood, Niranjan Naranapur Anand, Raghotham Hulgi, Juluri Suresh, Sonal Khare, Saravanakumar Dhakshinamoorthy

\section{Supplementary information}

\section{Generation and characterization of undifferentiated iPSCs from fibroblasts}

The iPSCs were derived from the fibroblasts as per the flow scheme shown in Figure S1 below. In brief, the culture plate was coated with attachment factor $(0.2 \%$ gelatin; Thermo: S006100) for $1 \mathrm{~h}$ followed by platting of MEF at $0.3 \times 10^{6}$ cells/well on day -2 . On day -1 , HDFn cells (Life Technologies: C-004-5C), at a density of $0.25 \times 10^{5}$ cells/well were seeded onto the 6 well plate. At day 0, the medium was changed, followed by transfection of HDFn cells with Epi5 vector (Invitrogen ${ }^{\mathrm{TM}}$ : A15960). On day 1, the cells were replaced with DMEM/F12 with HEPES supplemented with N-2, B-27, MEM NEAA, Gluta-MAX, $\beta$ mercaptoethanol, and bFGF (N2B27 medium). Established HDFn cells media was replaced every alternate day with fresh, N2B27 supplemented with FGF till day 15 . On day 16, the media was changed to complete Essential 8TM media (Gibco: A1517001) and was retained till day 25. The appearance of colonies was monitored during this process. The colonies showing iPSC morphology were stained for TRA-1-60 (Invitrogen ${ }^{\mathrm{TM}}$ : A25618), and these positive colonies were transferred to a 24 well plate pre-coated with vitronectin. The colonies were cryopreserved in liquid nitrogen until use. These frozen iPSCs were revived and checked for retention of morphology post freeze thawing.

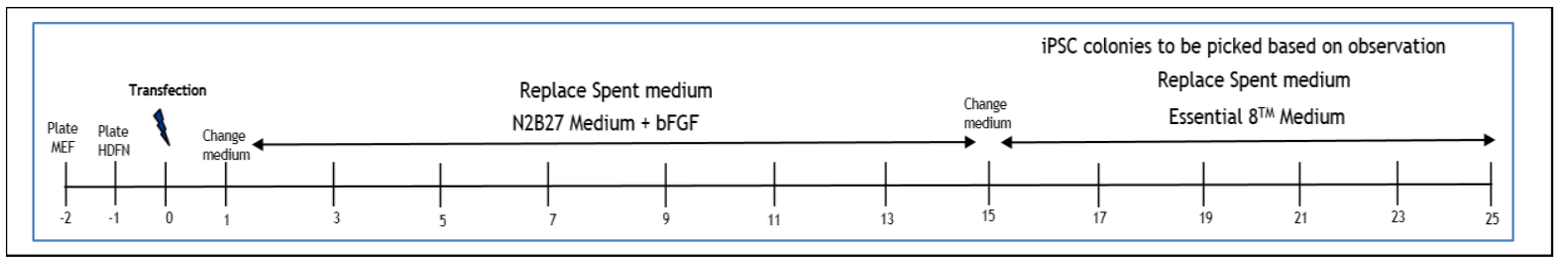

Figure S1. Scheme on generation of iPSC colonies from fibroblasts cells. 

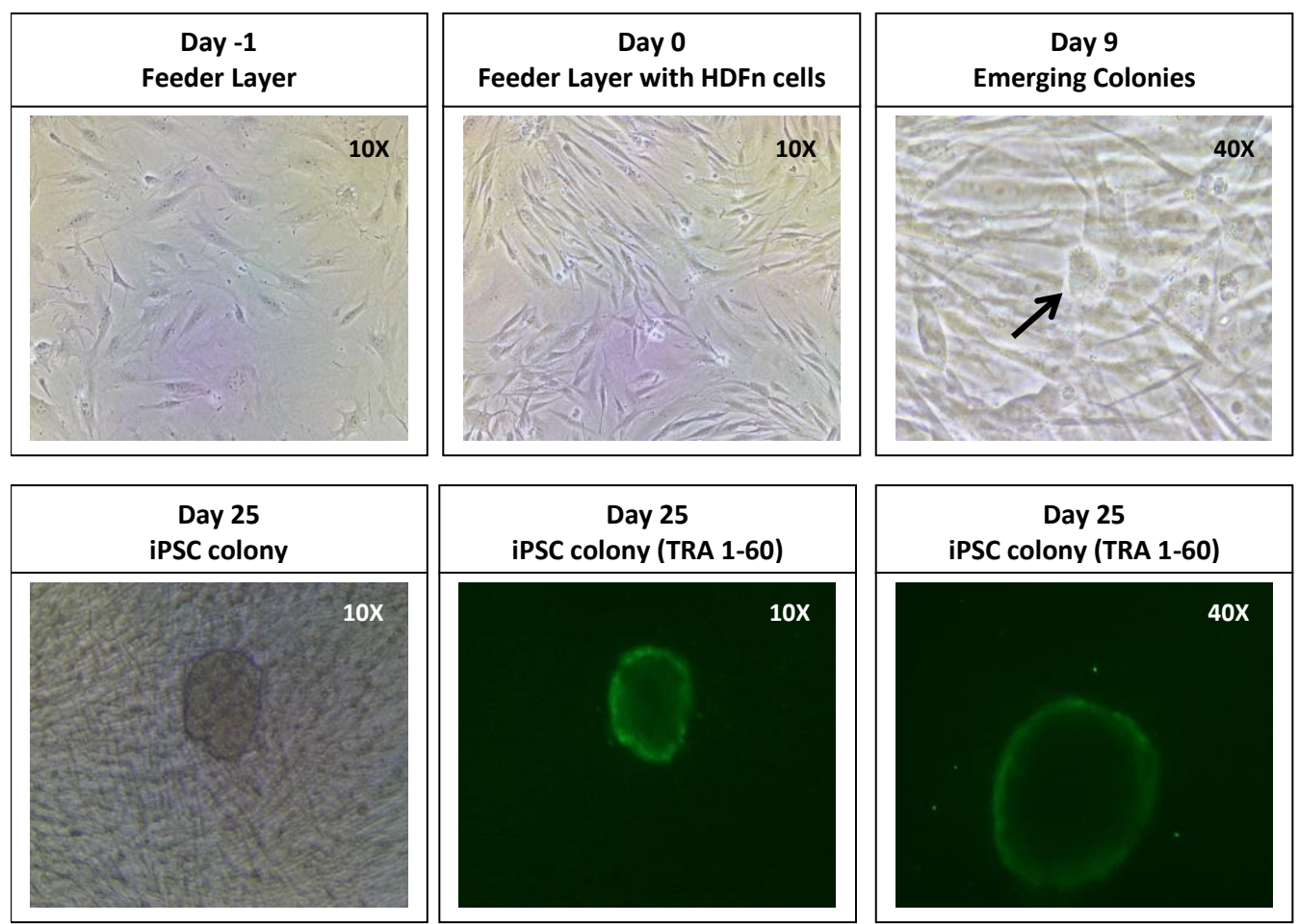

Figure S2. Live cell imaging and staining of iPSCs. Feeder layer was cultured on day -1, followed by seeding HDFn cells on day 0. The colonies emerged on day 9 and were maintained until day 25. These colonies were stained with TRA 1-60 - a marker for pluripotency. Briefly, $20 \mu \mathrm{L}$ of TRA-1-60 Alexa Fluor ${ }^{\mathrm{TM}} 488$ Conjugate (Invitrogen: A25618) in 1:50 dilution was added directly to the cell culture medium for staining. Culture media was mixed by gentle swirling. Incubation was carried out for 30 minutes at $37^{\circ} \mathrm{C}$. The staining solution was removed and the cells were gently washed for 2-3 times with PBS. Images of the cells were captured at various stages of the study.
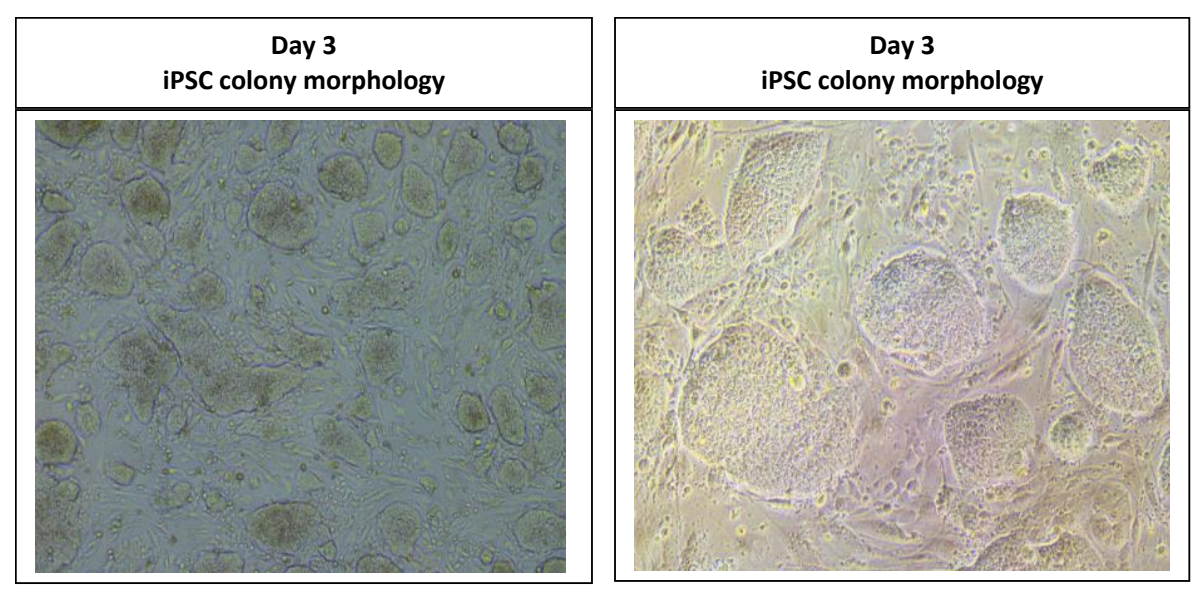

Figure S3. Morphology of iPSCs was retained upon freeze/thawing. The cultured iPSC colonies were frozen in $10 \%$ FBS and thawed to ascertain the efficiency of these 
cells/colonies to retain their parental morphology. The cells were thawed and seeded onto MEF layer cells. Day 3 pictures are shown in $5 \mathrm{X}$ and 10X magnification.

\section{Characterization of the iPSC colonies using pluripotency marker analysis by Q-PCR}

Total RNA was extracted from iPSCs using TrizolTM (Invitrogen) according to the manufacturer's instructions. The concentration of RNA was determined spectrophotometrically and the integrity of the RNA was assessed using a NanoDrop ${ }^{\circledR}$ ND1000 Spectrophotometer. $500 \mathrm{ng}$ of total RNA was used to prepare the cDNA samples. iScript $^{\mathrm{TM}}$ cDNA Synthesis Kit (BioRad: 1708890) was used for the cDNA synthesis and aliquots of cDNA's were stored at $-20^{\circ} \mathrm{C}$. Quantitative PCR (qPCR) analysis was performed using iTaq ${ }^{\mathrm{TM}}$ Universal SYBR ${ }^{\circledR}$ Green Supermix (BioRad: 172-5120) as per manufacturer's instructions. The cycling conditions included a denaturing step at $95^{\circ} \mathrm{C}$ for 2 min and 40 cycles of $95^{\circ} \mathrm{C}$ for $30 \mathrm{sec}, 60^{\circ} \mathrm{C}$ for $30 \mathrm{sec}$ and $45 \mathrm{sec}$ at $72^{\circ} \mathrm{C}$. Bio-Rad ${ }^{\circledR} \mathrm{CFX} 96^{\mathrm{TM}}$ was used to determine the expression level of selected target genes. Human $\beta$-Actin was used as control for normalization. Primer sequences used for the analysis are detailed in Table S1.

Table S1. Primers for characterization of iPSCs.

\begin{tabular}{|l|l|l|}
\hline \multicolumn{1}{|c|}{ Gene } & \multicolumn{1}{|c|}{ Forward Primer } & \multicolumn{1}{c|}{ Reverse Primer } \\
\hline KLF4 & GATGATGCTCACCCCACCTT & GGCGAATTTCCATCCACAGC \\
\hline SOX2 & TTTGTCGGAGACGGAGAAGC & TAACTGTCCATGCGCTGGTT \\
\hline OCT4 & GGCCACACGTAGGTTCTTGA & ATACCTTCCCAAATAGAACCCC \\
\hline 1-MYC & GGCCCCCAAAGTAGTGATCC & TGTCCAGACTGTCCCACCAT \\
\hline NANOG & AATGGTGTGACGCAGGGATG & GGACTGTTCCAGGCCTGATT \\
\hline TERT & CCTGCTCAAGCTGACTCGACACCGTG & GGAAAAGCTGGCCCTGGGGTGGAGC \\
\hline REX1 & CAG ATC CTA AAC AGC TCG CAG AAT & GCG TAC GCA AAT TAA AGT CCA GA \\
\hline LIN28 & GGTTCGGCTTCCTGTCCATGA & GGTGGCAGCTTGCATTCCTTG \\
\hline DPPA4 & ACACTGACAACCCCAGACCT & ACACACCACCTTTTGATTTGGGTA \\
\hline
\end{tabular}




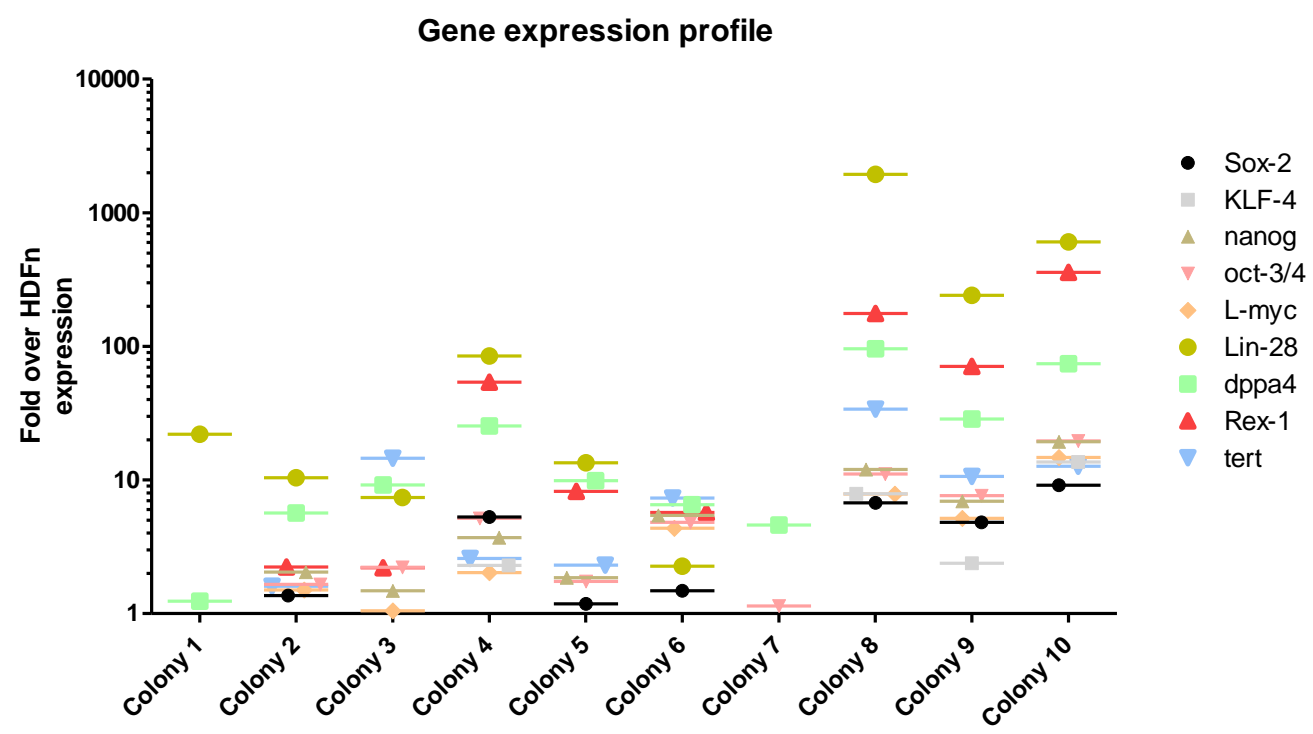

Figure S4. Gene expression analysis of the pluripotency markers from the iPSC colonies using RT-PCR. Colonies 4, 8, 9, and 10 showed the expression of all the pluripotency markers.

Table S2. Pluripotency marker analysis using RT-PCR. The colonies were scored for the respective genes based on the observed expression levels. Colonies 4, 8, 9 and 10 showed a perfect score of 9/9. These colonies were deemed positive for Yamanaka factors and pluripotency.

\begin{tabular}{|l|c|c|c|c|c|c|c|c|c|c|}
\hline & \multicolumn{9}{|c|}{ Yamanaka factors } \\
\hline Colony \# & Sox-2 & KLF-4 & oct-3/4 & L-myc & nanog & Lin-28 & dppa4 & Rex-1 & tert & scores \\
\hline Colony 1 & - & - & - & - & - & + & - & - & - & 1 \\
\hline Colony 2 & - & - & - & - & + & + & + & + & - & 4 \\
\hline Colony 3 & - & - & + & - & - & + & + & + & + & 5 \\
\hline Colony 4 & + & + & + & + & + & + & + & + & + & 9 \\
\hline Colony 5 & - & - & - & - & - & + & + & + & + & 4 \\
\hline Colony 6 & - & - & + & + & + & + & + & + & + & 7 \\
\hline Colony 7 & - & - & - & - & - & - & + & - & - & 1 \\
\hline Colony 8 & + & + & + & + & + & + & + & + & + & 9 \\
\hline Colony 9 & + & + & + & + & + & + & + & + & + & 9 \\
\hline Colony 10 & + & + & + & + & + & + & + & + & + & 9 \\
\hline
\end{tabular}



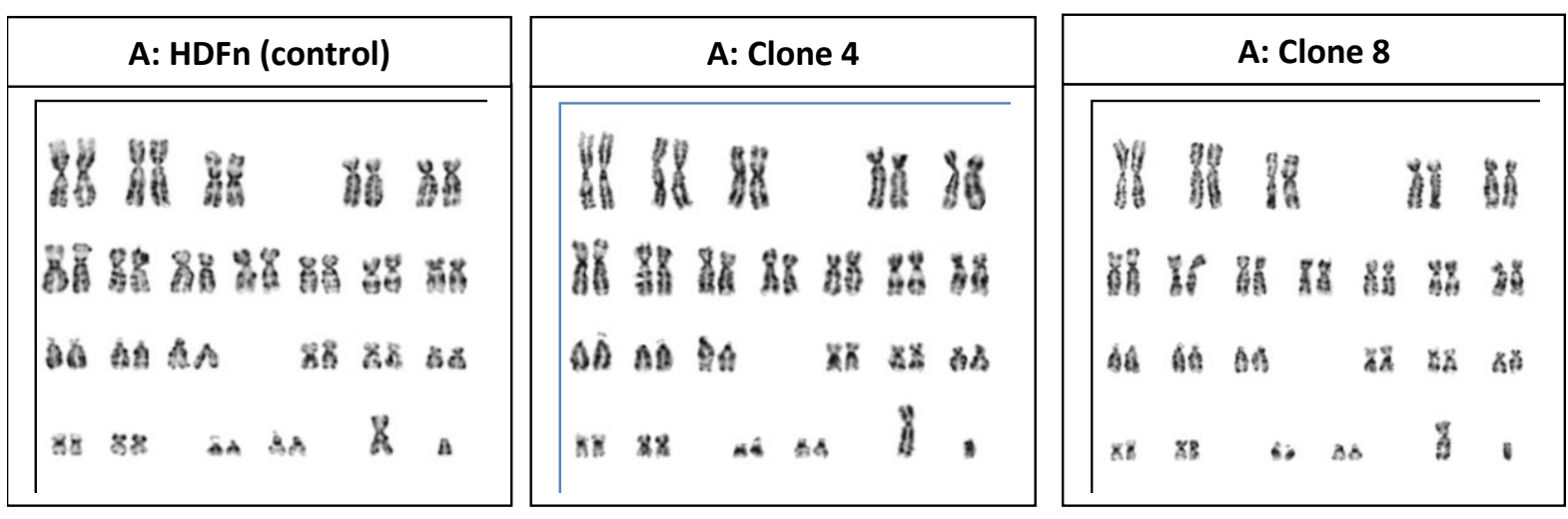

Figure S5. Characterization of iPSC lines via karyotyping. Genome integrity was analyzed using molecular cytogenetic technique. The genomic stability and integrity was assessed at Anand Diagnostic Laboratory (NABL-15189 accredited, Certificate. No: M0007), Bengaluru, India. Colonies 4 and 8 were found maintain the genome integrity. Colony 4 (clone 4) was used for all our experimentation.
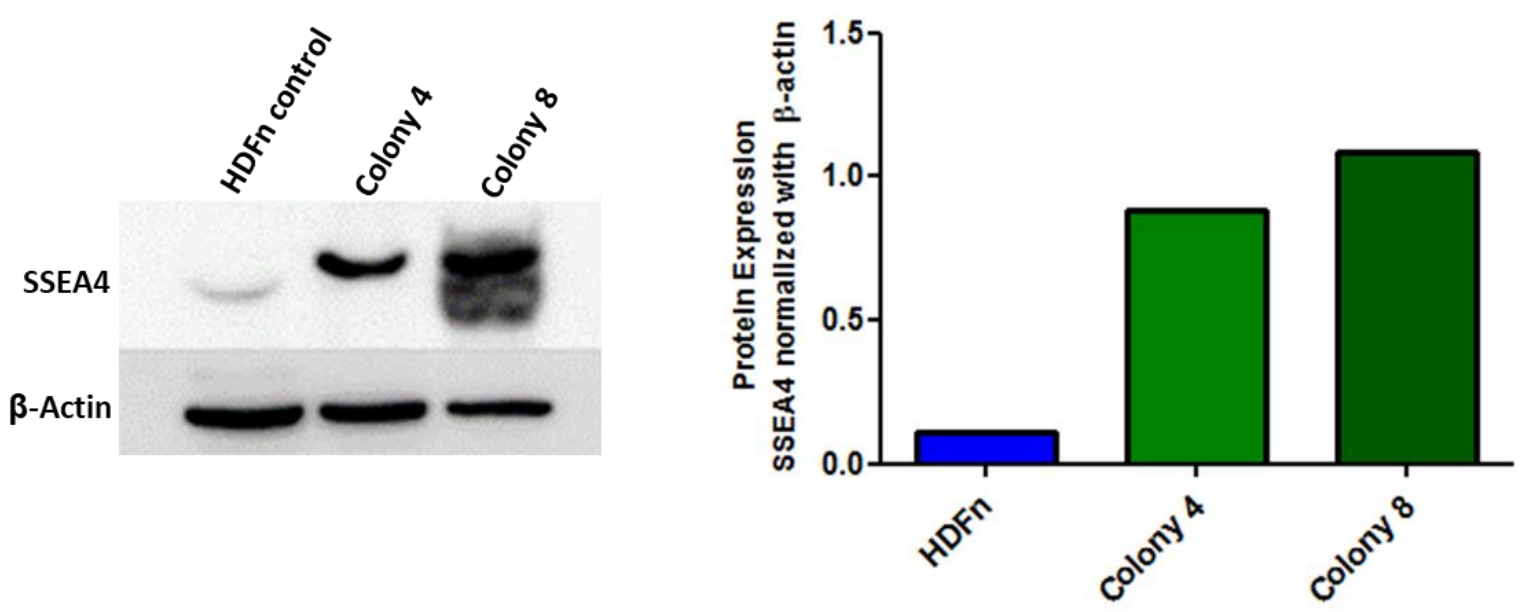

Figure S6. Western blot analysis for pluripotency marker. The expression level of the pluripotency marker, SSEA4 was analyzed in iPSC colonies 4 and 8 . The total proteins were extracted using RIPA buffer (Cell Signaling Technology: 9806). The protein samples were quantified using Bradford reagent according to manufactures instructions. $30 \mu \mathrm{g}$ protein was subjected to $8 \%$ sodium dodecyl sulfate polyacrylamide gel electrophoresis (SDS-PAGE), and transferred onto a nitrocellulose membrane. The membrane was incubated with purified anti human SSEA-4 antibody (Biolegend: 330401), followed by incubation with the secondary antibody coupled to horseradish peroxidase. The bands were visualized using LuminataTM Forte Western HRP Substrate (Millipore: WBLUF0100) in Image Quant LAS 4000 (GE Healthcare Life Sciences) and analyzed using Multi Gauge Ver. 2.0. The expression level of SSEA4 was normalized against $\beta$-Actin and plotted as a bar graph. 

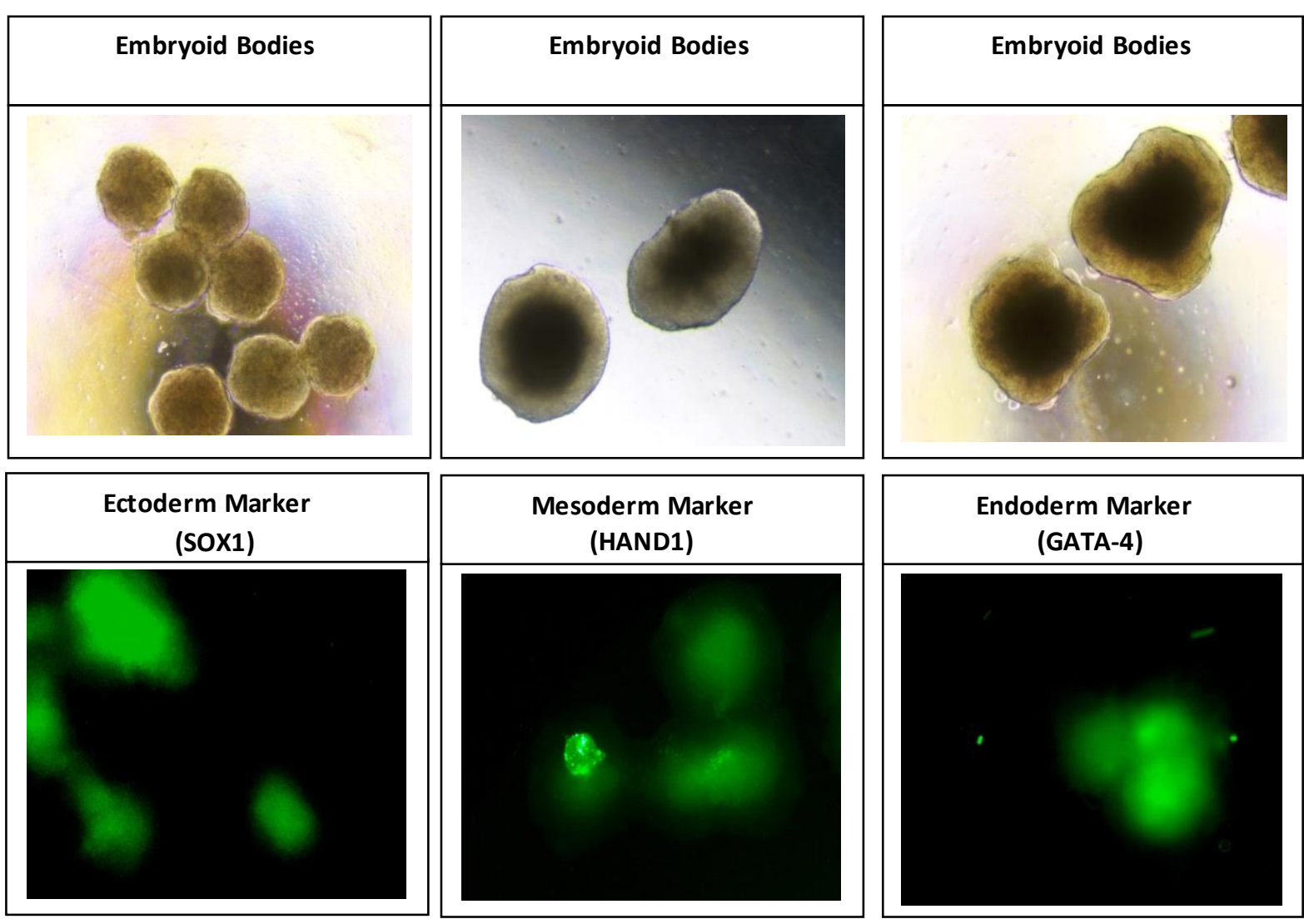

Figure S7. Embryoid body formation and differentiation into germ layers. The iPSCs were differentiated into Embryoid bodies using Hanging drop method and lineage towards germ layers (Ectoderm, Mesoderm, Endoderm) were characterized. Briefly, two days prior to EB formation, 60-70\% confluent iPSCs were passaged into vitronectin coated 6 well plates. On the day of EB formation, cells were washed once and then incubated with EDTA/PBS for 3-15 minutes to dissociate colonies to single cells and re-suspended in E8 media supplemented with PVA (Sigma: P8136) and ROCK inhibitor (Gibco: A2644501). Hanging drop experiment was initiated with single cell drops $(2000$ cells $/ 20 \mu \mathrm{L})$ cultured on the lid of the Petri dish. The culture was incubated in the dish for 2 days at $37^{\circ} \mathrm{C}$. The EB aggregates were washed and cultured further in poly-HEMA (Sigma: P3932) coated petri dish. The E8 medium was changed on alternate days until day 9 , followed by addition of differentiation medium (E6). Embryoid bodies were assessed for their function by directing them towards the formation of 3 germ layers - Endoderm, Mesoderm, and Ectoderm, which was observed on day 14 in the presence of E6 differentiation medium. A total of six markers were analyzed [ectoderm (OTX2 \& SOX1), mesoderm (Brachyury \& HAND1), and endoderm (GATA-4 \& SOX17)] and the representative images of one marker from each of ectoderm, mesoderm and endoderm are shown (SOX1, HAND1 and GATA-4 respectively). 
Characterization of the differentiated HLC using hepatic marker analysis by Q-PCR

Table S3. Primers for the characterization of HLC.

\begin{tabular}{|l|l|l|}
\hline \multicolumn{1}{|c|}{ Genes } & \multicolumn{1}{|c|}{ Forward Primer } & \multicolumn{1}{c|}{ Reverse Primer } \\
\hline $\begin{array}{l}\text { Fibrinogen alpha } \\
\text { chain (FGA) }\end{array}$ & $\begin{array}{l}\text { GTCTCGTATTAGAATTGTCACCC } \\
\text { A }\end{array}$ & TGCAAAGGGGATTTTCCTCA \\
\hline $\begin{array}{l}\text { Fibrinogen gamma } \\
\text { chain (FGG) }\end{array}$ & ACCAAGGTGGCACTTACTCA & CGGTCTTTTAAACGTCTCGAGC \\
\hline SOX17 & GACCGCACGGAATTTGAACA & GCTGCGGGGAGATTCACAC \\
\hline $\begin{array}{l}\text { Alpha - fetoprotein } \\
\text { (AFP) }\end{array}$ & $\begin{array}{l}\text { GAGAAGTACGGACATTCATGAAC } \\
\text { AA }\end{array}$ & TGCTGCCTTTGTTTGGAAGC \\
\hline Albumin (ALB) & CCCACGCCTTTGGCACAAT & ATCTCGACGAAACACACCCC \\
\hline
\end{tabular}

Total RNA was extracted from iPSCs and the differentiated HLC's using TrizolTM (Invitrogen) according to the manufacturer's instructions. The concentration of RNA was determined spectrophotometrically and the integrity of the RNA was assessed using a NanoDrop® ND-1000 Spectrophotometer. $500 \mathrm{ng}$ of total RNA was used to prepare the cDNA samples. iScript ${ }^{\mathrm{TM}}$ cDNA Synthesis Kit (BioRad: 1708890) was used for the cDNA synthesis and aliquots of cDNA's were stored at $-20^{\circ} \mathrm{C}$. Quantitative PCR (qPCR) analysis was performed using iTaq $^{\mathrm{TM}}$ Universal SYBR ${ }^{\circledR}$ Green Supermix (BioRad: 172-5120) as per manufacturer's instructions. The cycling conditions included a denaturing step at $95^{\circ} \mathrm{C}$ for 2 min and 40 cycles of $95^{\circ} \mathrm{C}$ for $30 \mathrm{sec}, 60^{\circ} \mathrm{C}$ for $30 \mathrm{sec}$ and $45 \mathrm{sec}$ at $72^{\circ} \mathrm{C}$. Bio-Rad ${ }^{\circ}$ CFX96 ${ }^{\mathrm{TM}^{\mathrm{M}}}$ was used to determine the expression level of selected target genes. Human $\beta$-Actin was used as control for normalization. Primer sequences used for the analysis are detailed in Table S3.

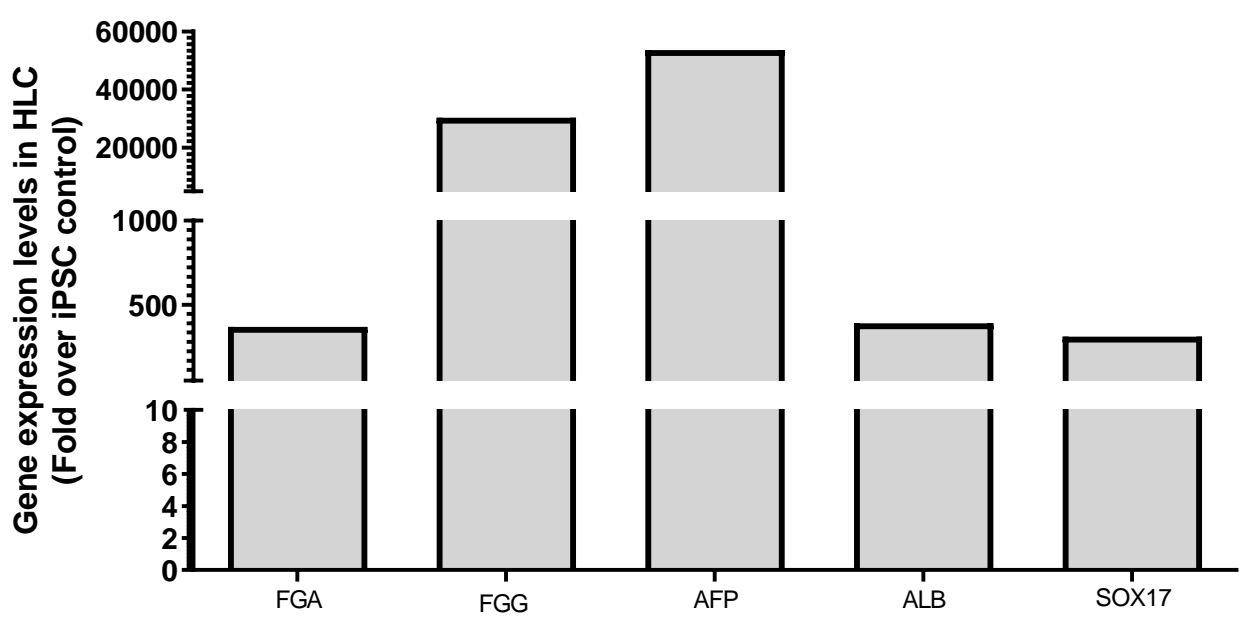

Figure S8. Gene expression analysis of the hepatic markers from HLC using Q-PCR. The hepatic genes such as FGA, FGG, AFP, ALB and SOX17 are highly expressed in HLC's. 